\title{
Design and Characterization of Non-planar 3D-printed Passive UHF-RFID Tag
}

\author{
N. Vidal ${ }^{1}$, A. Salas-Barenys ${ }^{1}$, A. Garcia ${ }^{1}$, J. Romeu$^{2}$, G. González², LL. Jofre², J. M. Lopez-Villegas ${ }^{1}$ \\ ${ }^{1}$ Department of Electronic and Biomedical Engineering, University of Barcelona (UB), Barcelona, Spain \\ ${ }^{2}$ Department of Signal Theory and Communications, CommSensLab, Universitat Politecnica de Catalunya (UPC), Barcelona, Spain \\ Email: nvidal@ub.edu
}

\begin{abstract}
This paper presents the design and preliminary characterization of a novel 3D passive UHF-RFID tag. The prototype is fabricated using additive manufacturing techniques: 3D printing and copper electroplating. The design, manufacturing process and measurement set-up are presented and discussed in detail. We propose a biconical antenna design with helical strips in the cones to provide compactness without breaking the symmetry of the component and to improve bandwidth. The antenna is matched to a commercial UHF-RFID integrated circuit. The good agreement between results and simulations allows us to validate the whole process.
\end{abstract}

\section{Keywords-3D printing, UHF RFID tag, antenna design}

\section{INTRODUCTION}

Since Charles W. Hull invented stereolithography in 1983, the potential of 3D printing technologies has increased year after year. In the context of electronic circuits and systems, 3D printing allows us to escape from planarity and could provide improved performance as well as increased compactness of electronic devices. In our previous works we analyzed nonplanar electronic design of 3D conical inductors and helicalmicrostrip transmission lines [1-2]. In this work we aim to explore the viability of fully $3 \mathrm{D}$ printing of small antennas. The goal is to assess the improvement in compactness and electrical performance related to the additional degree of freedom of a fully 3D design approach. As an example, we present a 3D-printed passive radio-frequency identification (RFID) tag. The 3D nature of the proposed prototype makes it suitable for fabrication using additive manufacturing (AM) techniques.

There are many research groups working on 3D printing technology for antenna applications. Some of these works were recently published in a special issue on "3D printed antennas and electromagnetic structures" [3]. If we refer to RFID technology, it has been widely used in many industrial applications for tracking and tracing industrial supply chains, textiles, pharmaceuticals, etc. An antenna design review for RFID tags is presented in [4] and a comparison of different fabrication techniques currently in use is described in [5]. Usually, tags are planar and use meanders to compact the antenna as much as possible. However, each meander introduces a discontinuity in the current path that must be considered in the design phase.

With 3D printing techniques we can explore non-planar models that may allow compactness without introducing any discontinuity and while maintaining the electrical performance. We intended to explore this option by designing a 3D helical antenna.

In order to miniaturize the device and enhance the tag range, instead of LF or HF bands, the proposed antenna operates at the UHF band. The UHF band also enlarges the

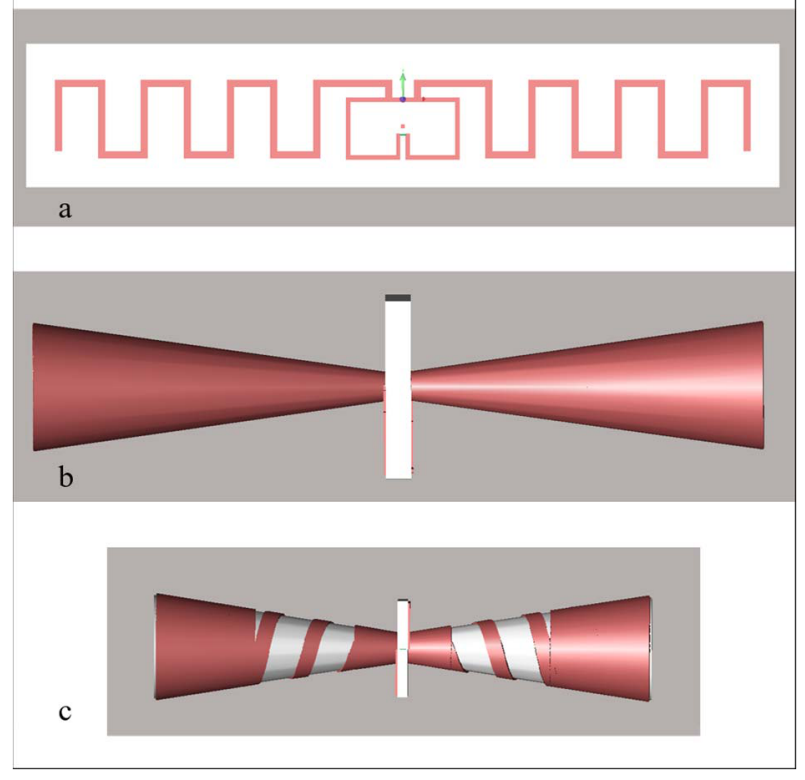

Fig. 1. CAD models of the RFID prototypes; (a) 2D tag, (b) 3D biconical tag and (c) 3D helical tag

operating bandwidth, improving the privacy and security of the communication protocols.

The article is organized as follows. In section II we explain the methodology, including: the antenna design procedure, the manufacturing process and the measurement set-up. In section III, the obtained results are described in detail. Electromagnetic (EM) simulation using the finite element method (FEM), as well as experimental results are presented and discussed. Finally, we present our conclusions and future trends in Section IV.

\section{Design And Manufacture of Antennas}

To perform the transition from a $2 \mathrm{D}$ planar tag to a $3 \mathrm{D}$ helical design, three different antennas were designed, manufactured and tested. For comparison with the commercial tags, first, a 2D meander-type antenna was designed. Then, the $2 \mathrm{D}$ to $3 \mathrm{D}$ transition was achieved with a biconical antenna. Finally, keeping the conical shape, a 3D helical antenna was proposed.

\section{A. Design of Antennas}

A view of the computer-assisted design (CAD) models of the antennas is shown in Fig. 1. For all the prototypes the base dielectric material is a photopolymer whose EM properties are a relative permittivity of 2.8 and a loss tangent of 0.02 . 
EM simulations were performed using the built-in FEM tool available in the EMPro design environment from Keysight Technologies Inc. The response of the antennas, in terms of the differential reflection coefficient and far field response was evaluated in the frequency range from $700 \mathrm{MHz}$ to $1 \mathrm{GHz}$.

All the prototypes were designed to match the input impedance of the Alien Higgs4 integrated circuit (IC) [6], which offers basic data for the tracking of resources. To compensate for the capacitive behavior of the IC, a double loop inductive matching network was implemented. For the $2 \mathrm{D}$ prototype, the matching network lies on the same plane as the radiators, while for the $3 \mathrm{D}$ prototypes it is defined in a perpendicular plate in the middle of the component.

In the case of the 3D helical antenna, radiators were defined on the external surface of the cones and consist of an outer conical section and an inner conical spiral (i.e., helicoids). Both helicoids were connected by means of a via hole in the lower edge of the matching network (Fig. 2). As can be seen in the figure, the network consists of metal loops on both sides of the plate. For manufacturing purposes, which will be discussed in the next section, all the metal parts were buried at half thickness of the dielectric material.

\section{B. Manufacture of Antennas}

The first steps in the manufacturing process were performed in the EMPro design environment. The flow chart is depicted in Fig. 3. The base 3D geometry of the antenna was created by combining the dielectric main body and the metal parts. After adding simulation ports and sensors, the antenna model was simulated and adjusted to obtain the desired specifications in terms of the reflection coefficient, at 866 $\mathrm{MHz}$. Once the optimized antenna design was obtained, all the metal parts were merged and subtracted from the dielectric main body to produce a printable 3D model.

An example of the printable 3D model for a helical antenna is shown in Fig. 4. It is worth noting that the metal parts turn into trenches and via holes on the surface of the main geometry. This is a consequence of the half thickness burial of metal parts, as mentioned in the previous section. Finally, the model was coded in an appropriate CAD format suitable for $3 \mathrm{D}$ printing.

The next steps of the manufacturing process are depicted in the flow chart of Fig. 5. First, the CAD file was sent to the printer. We used an Objet 260 Connex-1 multi-material jetting $3 \mathrm{D}$ printer from Stratasys. AM by material jetting is accomplished by depositing small drops of photopolymer material with spatial control, that is immediately cured using Ultraviolet (UV) light. The print preparation converts the $\mathrm{CAD}$ design into the printing instructions for each layer. The parts are built by depositing droplets of material from an inkjet printhead, forming each consecutive cross-section of the 3D antenna. Parts have a fine surface finish and require moderate post-processing. Supports are built from a secondary dissolvable material and they are removed manually using a water-jet system.

Once the 3D-printed parts were ready, we prepared the structure for electroless plating. Electroless plating requires a conductive seed for metal deposition and layer growing. In order to define that seed, the antenna's base geometries were covered in a conductive ink, then the areas without metallic parts were polished to remove the conductive coating.

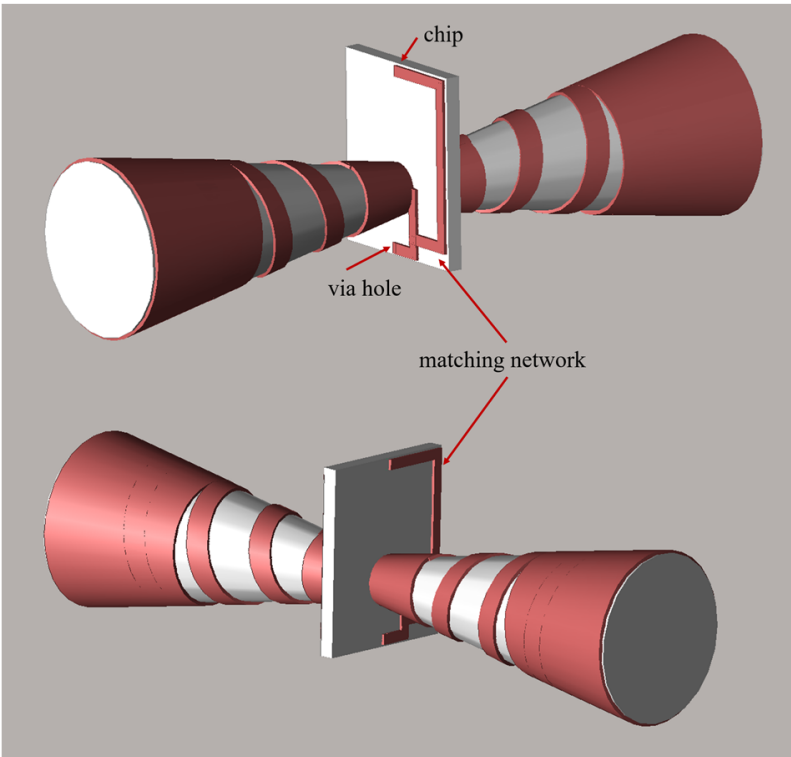

Fig. 2. 3D view of the helical antenna design. The matching network located at each side of the perpendicular plate is shown. The via hole and chip locations are included.

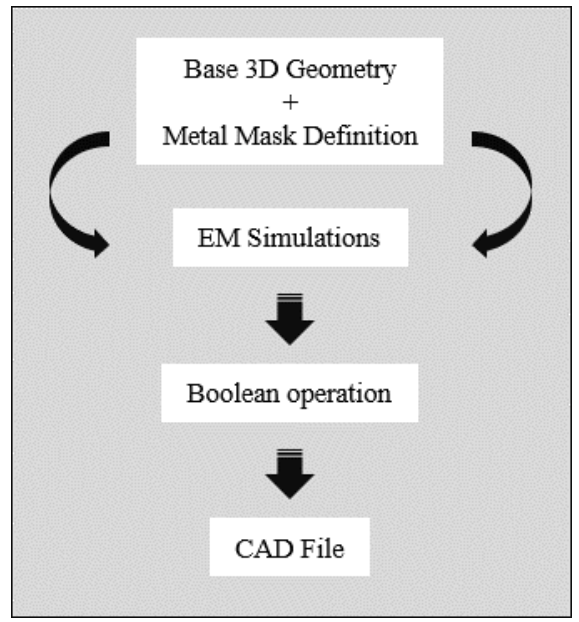

Fig. 3. Block diagram of the process flow for the manufacture of printed antennas (virtual phase).

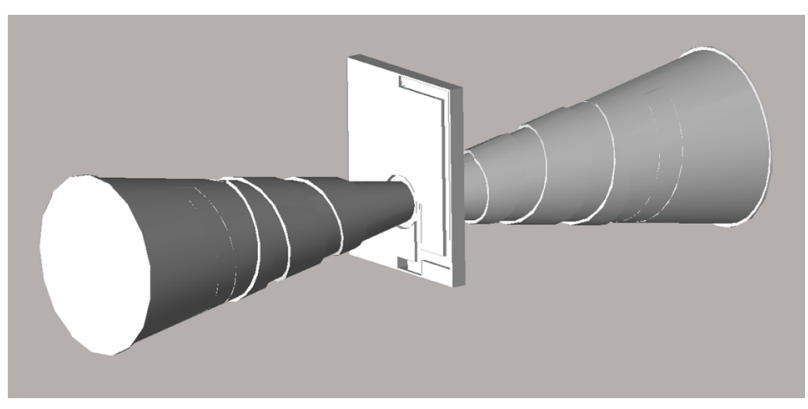

Fig. 4. View of the final helical antenna CAD file. 


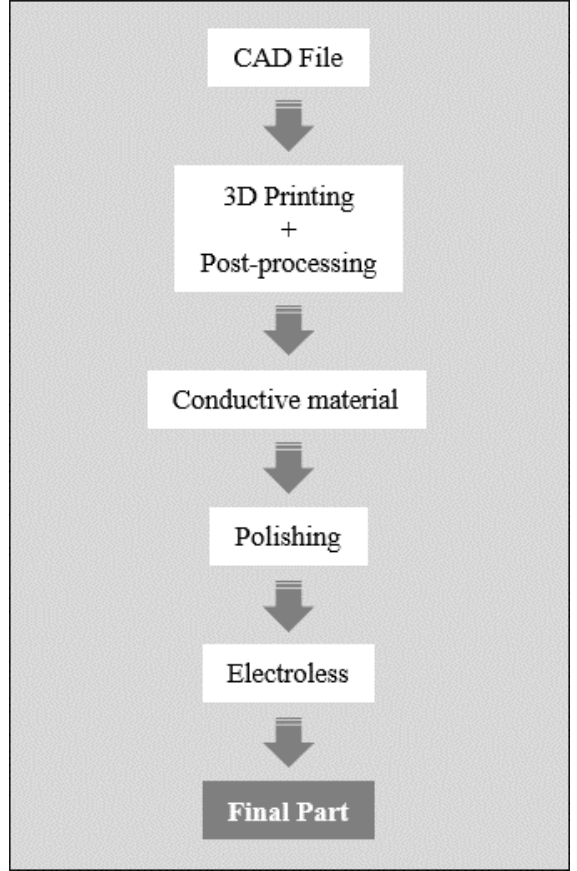

Fig. 5. Block diagram of the process flow for the manufacture of printed antennas (physical phase).

Accordingly, the seed only remained in the trenches and via holes of the surface. This was the reason for subtracting the buried metal parts from the main body. Finally, the copper plating was performed.

The final 3D helical antenna is shown in Fig. 6, together with the $2 \mathrm{D}$ and biconical manufactured prototypes. A more detailed description of the fabrication process can be found in our previous work, based on 3D-printed conical inductors [1]

\section{Measurements Set-up}

To validate the design and manufacturing processes of the antenna prototypes we needed to compare FEM simulation and experimental results. To that end, we measured the differential reflection coefficient of the antennas using a 4port E5071C vector network analyzer from Keysight Technologies Inc.

A single-ended to differential converter was designed and manufactured using printed circuit board (PCB) technology. The converter uses a miniaturized balun from Mini-Circuits. The component is shown, attached to the antennas, in Fig. 6. An SMA connector was soldered to one end of the converter. The opposite end was connected to the antenna at the RFID chip location. In order to de-embed the effect of the SMA connectors from the measurements, a custom calibration kit was fabricated, using the same converted layout and the appropriate load terminations. Finally, the commercial "Alien ALR-9900+" [7] reader was used for communication link characterization of the antenna prototypes, incorporating the RFID chip.

\section{RESULTS}

Results show a satisfactory agreement between simulations and measurements for the three prototypes. The 2D meander antenna is comparable to similar shaped commercial tags. This allows us to validate the processes of
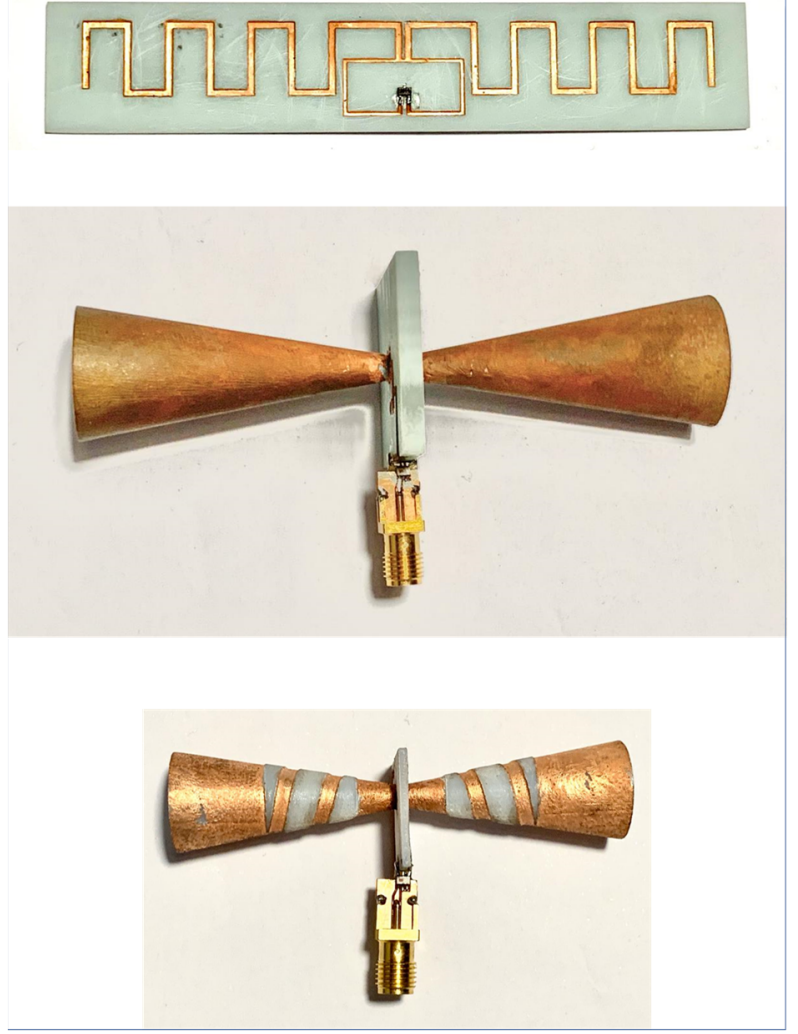

Fig. 6. View of the 2D and 3D antenna tags, mounted and ready for EM characterization.

metal deposition and layer growing. The biconical antenna allows us to test the whole 3D design and manufacturing process with a simple geometry before considering the helical metal strips in the cones. The measured reflection coefficient is in close agreement with the simulations results. This allows us to consider the helical antenna.

If we focus on the 3D helical antenna, we can observe how this geometric design reduces the size of the parts, leading to a more compact prototype. The final tag has a total length of $52 \mathrm{~mm}$ in front of the $105 \mathrm{~mm}$ of the 2D design and the $96 \mathrm{~mm}$ of the biconical antenna.

In Fig. 7 the simulated and measured reflection coefficients are represented. We observe a good agreement. The $-10 \mathrm{~dB}$ bandwidth is about $100 \mathrm{MHz}$ for the manufactured prototype. This bandwidth offers compensation for detuning effects due to the tolerances of the manufacturing process. Concerning the radiation properties of the antenna prototype, the system efficiency as a function of frequency is plotted in Fig. 8. A value of $93.69 \%$ is obtained at $866 \mathrm{MHz}$. Finally, Fig. 9 shows different cuts of the radiation pattern of the antenna. In contrast with 2D tag antennas, no asymmetries depending on the cut orientation are observed.

\section{CONCLUSION}

In this study we present analysis of the design, manufacture and performance of a non-planar 3D-printed passive UHF-RFID tag. Good agreement between simulation results and experimental measurements is observed. This 


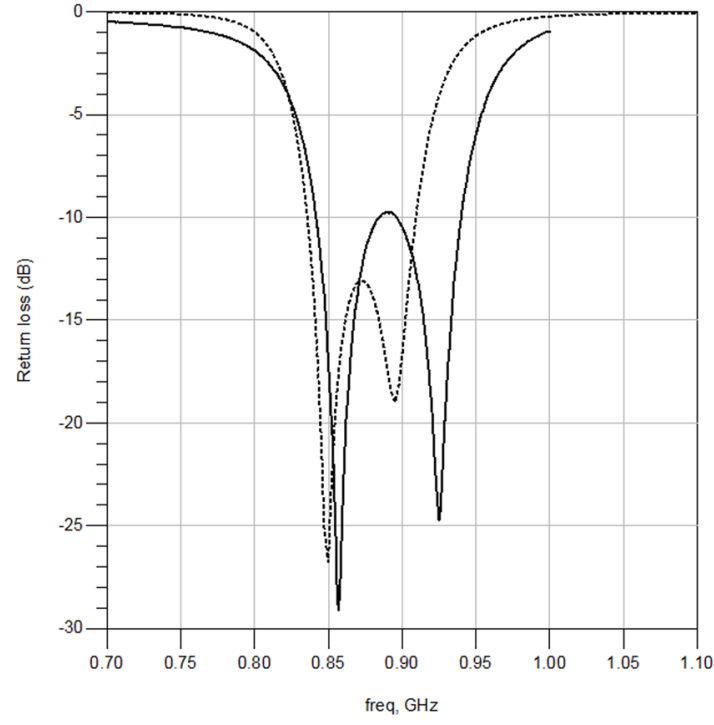

Fig. 7. Simulated (dotted line) and measured (continuous line) reflection coefficient of the $3 \mathrm{D}$ helical antenna tag.

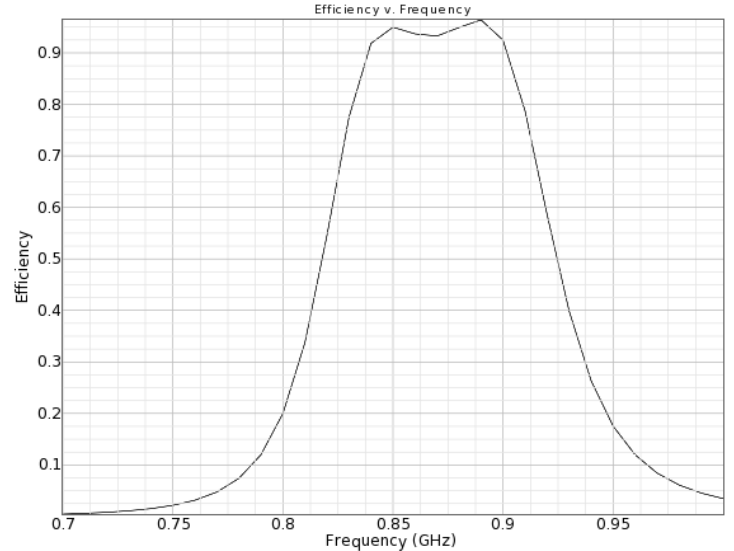

Fig. 8. System radiation efficiency of the $3 \mathrm{D}$ helical antenna tag.

agreement allows us to validate the whole design and manufacturing process. We propose a $3 \mathrm{D}$ helical geometry to obtain compactness of the complete device. The integration of the RFID IC into the antenna tag is considered. First, simulations were carried out to assess the impedance matching, the reflection coefficient, the gain and the operational range of the antenna. Then, the prototypes were fabricated using additive manufacturing (AM) techniques. Finally, we characterized the manufactured prototypes. The results demonstrate the feasibility of good-performance 3Dprinted antennas for RFID applications and show promising behavior of the tag for operation in the UHF-RFID band. Further research is needed to reduce the size. Reduced tag sizes may be of interest for different industrial applications. Based on the results, geometrical parameters such as the cone's angle, distance between metal strips, size of the strips, etc. should be analyzed to reduce the size of the prototypes while maintaining, as far as possible, the communication capabilities.

\section{ACKNOWLEDGMENT}

This work was supported by the Spanish Ministry of Economy and Competitiveness, project TEC2017-83524-R.
The authors would like to thank Alien Technologies for providing the IC.

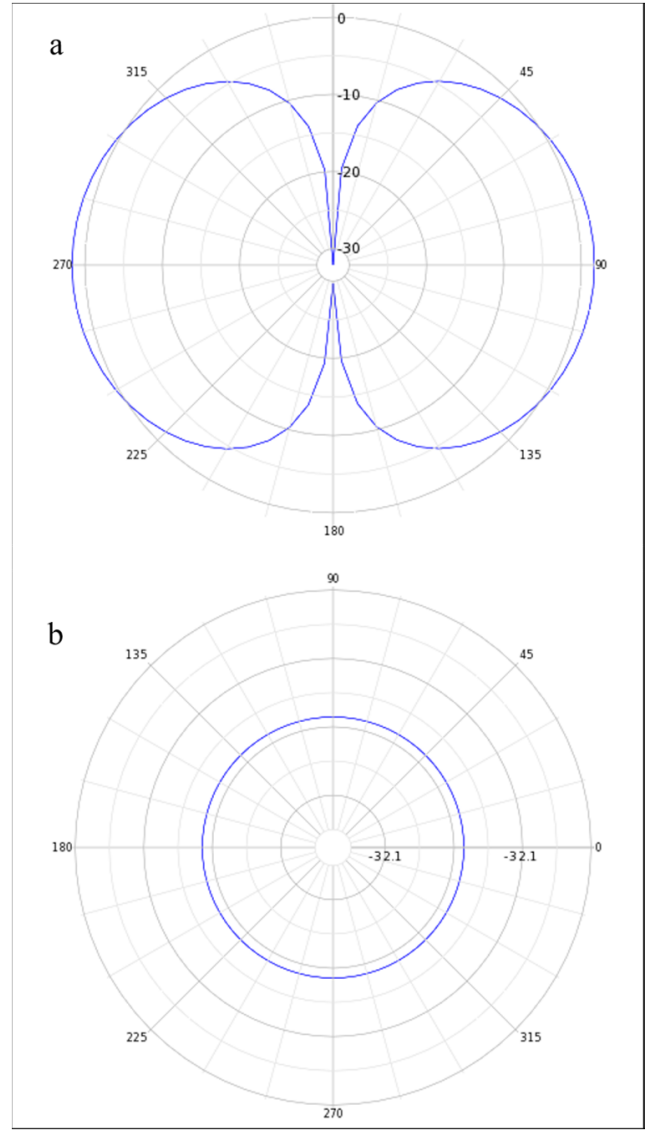

Fig. 9. a) Polar cut of the radiation gain as a function of theta for phi $=0^{\circ}$. b) Polar cut of the radiation gain as a function of phi for theta $=30^{\circ}$.

\section{REFERENCES}

[1] J. M. Lopez-Villegas, N. Vidal, J. Sieiro, A. Salas, B. Medina, and F. M. Ramos, "Study of 3-D printed conical inductors for broadband RF applications," IEEE Transactions on Microwave Theory and Techniques, vol. 66, no. 8, pp. 3597-3602, 2018.

[2] J. M. Lopez-Villegas, A. Salas, and N. Vidal, "Modeling of 3-d-printed helical-microstrip transmission lines for RF applications," IEEE Transactions on Microwave Theory and Techniques, vol. 67, no. 12, pp. 4914-4921, 2019.

[3] Sharma, SK, Xin, H, Wu, BI, Vardaxoglou, JC, Chan, "Guest Editorial Special Cluster on Three-Dimensional Printed Antennas and Electromagnetic Structures," IEEE Antennas and Wireless Propagation Letters, 17(11), pp.1998-2002, 2018.

[4] K. V. Seshagiri Rao, Pavel V. Nikitin, and S. F. Lam," Antenna Design for UHF RFID Tags: A Review and a Practical Application," IEEE Transactions on Antennas and Propagation, vol. 53, no. 12, pp. 38703876, 2005.

[5] R. Colella, A. Rivadeneyra, A. J. Palma, L. Tarricone, L. F. CapitánVallvey, L. Catarinucci, and J. F. Salmerón, "Comparison of Fabrication Techniques for Flexible UHF RFID Tag Antennas," IEEE Antennas and Propagation Magazine, 59(5), pp.159-168, 2017.

[6] "Higgs 4 RFID IC | Alien Technology.” [Online]. Available: http://www.alientechnology.com/products/ic/higgs-4/. [Accessed: 18March-2020].

[7] “Alien Technology Readers." [Online]. Available: http://www.alientechnology.com/products/readers/. [Accessed: 18March-2020] 